\title{
Tratamento Farmacológico da Síndrome de Abstinência Alcoólica
}

\author{
Pharmacological Treatment of Alcohol Withdrawal
}

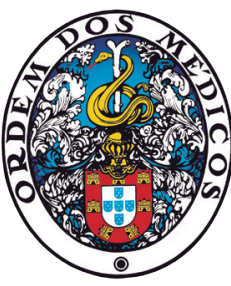

Joana TEIXEIRA $\triangle^{1}$

Acta Med Port 2022 Apr;35(4):286-293 - https://doi.org/10.20344/amp.15799

RESUMO

Introdução: Quando os consumidores excessivos crónicos de álcool, particularmente os indivíduos com o diagnóstico de perturbação de uso de álcool, suspendem abruptamente o consumo de bebidas alcoólicas, pode surgir um quadro clínico denominado síndrome de abstinência alcoólica. Nestes casos, é necessário muitas vezes instituir tratamento farmacológico para aliviar os sintomas e prevenir as complicações da abstinência. O tratamento farmacológico da síndrome de abstinência alcoólica é utilizado em unidades especializadas no tratamento de perturbação de uso de álcool, mas também pode ser necessário em serviços médico-cirúrgicos, quando esta síndrome se manifesta em doentes com perturbação de uso de álcool internados por patologias orgânicas.

Métodos: Revisão de guidelines disponíveis na literatura sobre tratamento da síndrome de abstinência alcoólica com o objetivo de determinar qual o tratamento farmacológico recomendado.

Resultados: Foram analisadas as quatro guidelines sobre tratamento farmacológico da síndrome de abstinência alcoólica (National Institute for Health and Care Excellence, American Society of Addiction Medicine, World Federation of Societies of Biological Psychiatry e American Psychiatric Association), em relação às suas recomendações e evidência da eficácia dos fármacos para tratamento da síndrome de abstinência alcoólica. Esta análise foi ainda complementada pela consulta das guidelines de Maudsley.

Conclusão: É fundamental manter um nível elevado de suspeita para o eventual aparecimento da síndrome de abstinência alcoólica em todos os contextos clínicos. Nesses casos, importa saber intervir precocemente.

Palavras-chave: Abstinência de Álcool; Alcoolismo/tratamento farmacológico; Síndrome de Abstinência

\section{ABSTRACT}

Introduction: Excessive chronic alcohol users, and particularly patients with alcohol use disorder, may present an alcohol withdrawal syndrome if they abruptly stop drinking. Alcohol withdrawal syndrome requires pharmacological treatment for the treatment of withdrawal symptoms and to prevent withdrawal complications. Medically assisted withdrawal treatment is used in alcohol treatment units, but it is also frequently required in patients admitted to hospital for other conditions.

Methods: Review of major guidelines covering treatment of withdrawal syndrome with the aim to describe recent evidence and recommendations about the pharmacological treatment for alcohol withdrawal syndrome.

Results: Four major guidelines concerning treatment of withdrawal syndrome were selected (National Institute for Health and Care Excellence, American Society of Addiction Medicine, World Federation of Societies of Biological Psychiatry and American Psychiatric Association) and screened for the recommendations and level of evidence on drug prescribing for alcohol withdrawal syndrome. The Maudsley guidelines were also considered in this review.

Conclusion: Maintaining a high level of suspicion for alcohol withdrawal syndrome is important across all clinical settings, and it should be promptly treated.

Keywords: Alcohol Abstinence; Alcoholism/drug therapy; Substance Withdrawal Syndrome

\section{INTRODUÇÃO}

Portugal está entre os países com maiores consumos de bebidas alcoólicas. ${ }^{1}$ Dados do 'Retrato da Saúde' publicado pelo Ministério da Saúde em 2018 mostram que $38,3 \%$ dos homens portugueses consumiam álcool diariamente, o valor mais alto da Europa, e que as mulheres portuguesas registaram o consumo diário de álcool mais elevado na União Europeia $(11,8 \%){ }^{2}$ O relatório de 2018 da Organização Mundial de Saúde (OMS) mostra que a prevalência das perturbações de uso do álcool (PUA) em Portugal é de $6,8 \%$ e a de dependência de álcool é de $3 \%{ }^{3,4}$

Os riscos associados aos consumos de bebidas alcoólicas vão aumentando de forma progressiva e em continuidade com a frequência e a intensidade das ingestões de álcool. É conhecido que o consumo excessivo crónico de álcool provoca alterações neuroquímicas a nível do sistema nervoso central.
Apesar de ser uma molécula simples, o etanol afeta vários sistemas de neurotransmissores cerebrais. O mecanismo de ação do álcool inclui efeitos no sistema gabaérgico, em que a ativação recorrente dos recetores GABA, resultante do consumo continuado de álcool, altera o normal equilíbrio GABA/glutamato. A longo prazo, este desequilíbrio conduz a um processo adaptativo de aumento dos níveis de glutamato, de modo a se alcançar um novo equilíbrio GABA/glutamato. ${ }^{5,6}$

Quando os consumidores excessivos crónicos de álcool, particularmente os indivíduos com diagnóstico de PUA, suspendem abruptamente o consumo de bebidas alcoólicas, pode surgir um quadro de privação de uso de álcool, denominada síndrome de abstinência alcoólica.

A síndrome de abstinência alcoólica, cujos critérios de diagnóstico se encontram descritos no DSM-5 e CID-10,

1. Unidade de Alcoologia e Novas Dependências. Centro Hospitalar Psiquiátrico de Lisboa. Lisboa. Portugal.

$\triangle$ Autor correspondente: Joana Teixeira. joanateixeira@chpl.min-saude.pt

Recebido/Received: 25/01/2021 - Aceite/Accepted: 14/05/2021 - Publicado Online/Published Online: 12/07/2021 - Publicado/Published: 01/04/2022 Copyright $\odot$ Ordem dos Médicos 2022 
inclui um amplo espectro de sintomas, variável de indivíduo para indivíduo, e surge tipicamente nas primeiras três a 12 horas após a suspensão, ou após uma marcada redução, dos consumos de álcool, podendo persistir durante quatro ou cinco dias. ${ }^{7,8}$

Os sintomas de privação alcoólica, resultantes de uma hiperatividade autonómica, incluem tremores, náuseas e vómitos, sudorese, ansiedade, insónia, taquicardia, elevação da tensão arterial e alterações de humor.

Em casos ligeiros de PUA, a síndrome de abstinência alcoólica pode remitir sem surgirem complicações e muitas vezes sem necessidade de tratamento farmacológico. No entanto, em casos moderados a graves de PUA, a síndrome de abstinência alcoólica poderá evoluir e complicar-se caso não tenha tido tratamento farmacológico adequado. As complicações mais frequentes da síndrome de abstinência alcoólica são as convulsões e o delirium tremens.

O delirium tremens é a complicação mais grave e mais perigosa da síndrome de abstinência alcoólica, sendo considerado uma emergência médica. ${ }^{9}$ Tem uma prevalência de cerca de $5 \%$ ( $3 \%$ - 15\%) em doentes que apresentam sintomas de privação de álcool. ${ }^{10}$ Geralmente dura entre 48 e 72 horas, mas pode persistir por um período de tempo superior. É mais frequente em doentes críticos, nos quais está recomendada a prescrição de tratamento farmacológico em doses elevadas para prevenção da síndrome de abstinência alcoólica. ${ }^{11}$ As manifestações mais frequentes do delirium tremens são a instabilidade autonómica, febre, desidratação, desequilíbrios hidro-eletrolíticos, hipoglicémia, insuficiência hepática, pancreatite, sépsis, meningite, hemorragia intracraniana e encefalopatia de Wernicke-Korsakoff. O controlo da agitação é fundamental em doentes com delirium tremens, devendo nessas situações reduzir-se os níveis de agitação enquanto durar o período de delirium tremens. ${ }^{8}$

O tratamento farmacológico da síndrome de abstinência alcoólica tem por objetivo aliviar os sintomas de privação, prevenir as complicações da abstinência de álcool e, quando inserido num programa de tratamento de uma PUA, dar início ao processo de reabilitação do indivíduo.

Se é expectável a necessidade de instituir tratamento farmacológico para prevenção da síndrome de abstinência alcoólica em doentes com PUA moderada ou grave que realizam desabituação física de álcool em regime de internamento em unidades especializadas, existem outros contextos em que esta necessidade não seria à partida previsível.

Sabe-se que a prevalência do uso de substâncias em doentes que recorrem aos serviços de urgência é elevada, sendo várias as especialidades médicas que são confrontadas com as complicações físicas e psiquiátricas da dependência de substâncias. ${ }^{12,13}$ Adicionalmente, os próprios sintomas, ou complicações dos quadros de privação de álcool, podem ser eles mesmos o motivo de recurso ao Serviço de Urgência. O rastreio para identificação de perturbações de uso de substâncias ainda não é rotina no serviço de urgência hospitalar e a prioridade de intervenção na urgência muitas vezes não passa pelo rastreio dos consumos de substâncias. ${ }^{14,15}$ No entanto, sendo os doentes com PUA utilizadores frequentes dos serviços de urgência, poderá ser necessário internar estes doentes em serviços de especialidades médicas ou cirúrgicas, por descompensação de patologias orgânicas associadas ou por lesões traumáticas agudas, muito frequentes nesta população. ${ }^{16}$

Adicionalmente, se for necessário internar um doente com PUA para realizar um tratamento médico-cirúrgico eletivo, dada a ausência de consumos de álcool durante o internamento, pode surgir uma síndrome de abstinência alcoólica com necessidade de intervenção farmacológica para aliviar os sintomas e prevenir o aparecimento de complicações da síndrome de abstinência alcoólica.

Assim, sempre que ocorra uma paragem não voluntária de consumos de álcool por parte do doente com PUA, e quando a PUA não é o motivo pelo qual o doente se encontra a receber tratamento hospitalar, é particularmente importante que os médicos estejam alerta e mantenham um elevado nível de suspeição para o eventual aparecimento de um quadro de síndrome de abstinência alcoólica e para a consequente necessidade de intervenção precoce. Nestes casos, deverá ser rapidamente instituída terapêutica indicada, de modo a aliviar os sintomas e prevenir as complicações da síndrome de abstinência alcoólica.

$\mathrm{Na}$ escolha da terapêutica a utilizar no tratamento da desintoxicação de álcool realizada a nível hospitalar, quer seja voluntária num internamento eletivo de desabituação em unidade de tratamento especializada, ou involuntária por necessidade de internamento num serviço médico ou cirúrgico para tratamento de patologia orgânica, há vários aspetos a considerar.

Este trabalho tem como objetivo determinar qual a terapêutica farmacológica recomendada no tratamento da síndrome de abstinência alcoólica.

\section{MÉTODOS}

Revisão de normas de orientação clínica (guidelines) disponíveis na literatura sobre o tratamento farmacológico da síndrome de abstinência alcoólica: guidelines do $\mathrm{Na}$ tional Institute for Health and Care Excellence (NICE), da American Society of Addiction Medicine (ASAM), da World Federation of Societies of Biological Psychiatry (WFSBP), e da American Psychiatric Association (APA) ${ }^{9,17-21}$ De realçar que na elaboração das guidelines da WFSBP foram revistas e incorporadas as evidências da Associação Alemã de Psiquiatria, Psicoterapia e Psicossomática (DGPPN), da Associação Inglesa de Psicofarmacologia, da Sociedade Francesa de Alcoologia e da Federação Europeia das Sociedades de Adictologia. ${ }^{9}$ A revisão foi complementada pela consulta das guidelines de Maudsley. ${ }^{5}$

\section{RESULTADOS}

Segundo as guidelines do NICE (outubro de 2020) para o tratamento das perturbações de uso de álcool, o tratamento farmacológico de primeira linha recomendado no tratamento da síndrome de abstinência alcoólica são as 
benzodiazepinas. ${ }^{17-19}$ Como segunda linha, em alternativa às benzodiazepinas, recomendam a carbamazepina e o clometiazol, devendo este último, no entanto, ser apenas utilizado com precaução e em meio hospitalar. ${ }^{17-19}$

No caso de aparecimento de complicações da abstinência de álcool, como delirium tremens e convulsões, as guidelines NICE também fazem algumas recomendações. Em situações de delirium tremens, o tratamento de primeira linha indicado é lorazepam por via oral. Caso haja persistência de sintomas ou recusa de toma de terapêutica por via oral, deverá ser utilizado lorazepam ou haloperidol por via parentérica. É igualmente recomendado que, em situações de delirium tremens em doentes que se encontrem a realizar tratamento para a síndrome de abstinência alcoólica, se proceda a uma revisão da terapêutica farmacológica prescrita. Na eventual ocorrência de convulsões por privação de álcool está recomendada a utilização de benzodiazepinas de semivida curta, como lorazepam. A utilização de fenitoína está contraindicada para tratamento das convulsões por privação de álcool. É igualmente recomendado que, em situações de convulsões em contexto de privação de álcool em doentes que se encontrem a realizar tratamento para a síndrome de abstinência alcoólica, se proceda a uma revisão da terapêutica farmacológica prescrita. ${ }^{17-19}$

As guidelines do NICE preconizam também a prescrição de tiamina em doentes com alto risco de desenvolver encefalopatia de Wernicke, ou nos casos suspeitos. A utilização de tiamina oral profilática está recomendada em doentes com PUA que se encontrem malnutridos ou em risco de má nutrição, na presença de doença hepática descompensada, ou durante o tratamento de desabituação de álcool medicamente assistida. A prescrição de tiamina profilática deve ser inicialmente realizada por via parentérica e posteriormente substituída pela prescrição de tiamina por via oral em doentes com PUA que estejam malnutridos ou em risco de má nutrição; que tenham doença hepática descompensada e que adicionalmente se encontrem num serviço de urgência; ou que se encontrem internados devido a patologia aguda. Em casos suspeitos de encefalopatia de Wernicke deve ser prescrita tiamina por via parentérica. É recomendado que se mantenha um elevado nível de suspeita da ocorrência de encefalopatia de Wernicke, particularmente em doentes com intoxicação aguda de álcool. A administração de tiamina por via parentérica deve ser mantida por um período mínimo de cinco dias, a menos que seja excluído o diagnóstico de encefalopatia de Wernicke. No final desse período deve ser prescrita tiamina por via oral. ${ }^{17-19}$

De acordo com as guidelines da American Society of Addiction Medicine (ASAM) de 2020, o tratamento para a síndrome de abstinência alcoólica diverge consoante seja realizado em regime de ambulatório ou de internamento. ${ }^{20}$ Em regime de internamento, as guidelines da ASAM recomendam as benzodiazepinas como fármacos de escolha em primeira linha, pela evidência bem documentada do seu efeito na redução dos sintomas de privação de álcool, incluindo na redução de incidência de convulsões e de de- lirium. De acordo com as recomendações da ASAM, a carbamazepina e a gabapentina também podem ser utilizadas em alternativa às benzodiazepinas em doentes com síndromes de abstinência de intensidade ligeira a moderada (pontuação inferior a 19 obtida na escala de avaliação de abstinência CIWA-AR). ${ }^{21} \mathrm{Em}$ doentes com contraindicação para a utilização de benzodiazepinas está recomendado o uso de fenobarbital, mas este último apenas deve ser prescrito por médicos com experiência na sua utilização. ${ }^{20}$

Quanto à escolha de benzodiazepinas, as guidelines da ASAM recomendam a opção por uma com semivida mais longa, embora ressalvem que não existe nenhuma benzodiazepina que tenha eficácia superior documentada relativamente às restantes. Enquanto se aguardam os resultados dos exames analíticos de avaliação da função hepática, ou em situações de ausência de disponibilidade para realizar essa avaliação, bem como em casos de doença hepática significativa, devem ser prescritas benzodiazepinas que não apresentam metabolização hepática, como o lorazepam e o oxazepam. É igualmente recomendada pela ASAM a utilização de doses mais reduzidas de benzodiazepinas em doentes com insuficiência hepática e em idosos. Para utilizar doses menos elevadas de benzodiazepinas poderá ser prescrita uma associação de antipsicótico com benzodiazepina. ${ }^{20}$

Nos casos em que há contraindicação para uso de benzodiazepinas, as guidelines da ASAM recomendam a prescrição de carbamazepina ou, em alternativa, de gabapentina, mas apenas para síndrome de abstinência alcoólica de intensidade ligeira a moderada. Contudo, ressalvam que não há evidência de superioridade de eficácia destas sobre outras benzodiazepinas no tratamento da síndrome de abstinência alcoólica. As guidelines da ASAM referem ainda que, como associação às benzodiazepinas, se poderá utilizar carbamazepina, gabapentina ou ácido valpróico no tratamento da síndrome de abstinência alcoólica. Alertam, porém, que não deverá ser prescrito ácido valpróico em doentes com doença hepática nem em mulheres em idade fértil. ${ }^{20}$

Outra alternativa apresentada pela ASAM, para situações em que está contraindicada a prescrição de benzodiazepinas, é o uso de fenobarbital. Porém, a ASAM alerta que apenas deverá ser prescrito fenobarbital em ambiente hospitalar por médicos com experiência na sua utilização, devido à sua estreita janela terapêutica e efeitos secundários. $^{20}$

As guidelines da ASAM recomendam que se utilizem fármacos beta-bloqueantes e agonistas alfa-2 adrenérgicos, tais como propanolol e clonidina, estritamente em associação com a prescrição de benzodiazepinas para controlo dos sinais de hiperatividade autonómica e da ansiedade quando estes não estão controlados apenas pela toma de benzodiazepinas. Os fármacos beta-bloquentes e agonistas alfa-2 adrenérgicos não deverão ser utilizados em monoterapia para prevenção ou tratamento da síndrome de abstinência alcoólica. A utilização de magnésio para profilaxia ou tratamento da síndrome de abstinência 
alcoólica não está recomendada pelas guidelines da ASAM mais recentes. Esta posição contraria as guidelines de 2004 em resultado da ausência de benefício comprovado da prescrição de magnésio para esse efeito demonstrada pelos dados obtidos numa recente revisão Cochrane. ${ }^{22}$ Atualmente, a prescrição de magnésio apenas está recomendada em doentes com hipomagnesiémia, arritmias cardíacas, desequilíbrios hidro-electrolíticos, ou em doentes com história prévia de convulsões em contexto de privação de álcool. ${ }^{20}$

É igualmente recomendado pelas guidelines da ASAM que, em situações de desidratação ou de desequilíbrios hidro-eletrolíticos por défice em ácido fólico, magnésio, fósforo ou potássio, se proceda à correção desses défices..$^{20,23}$

Em relação ao tratamento das complicações da síndrome de abstinência, as guidelines da ASAM recomendam, quer no tratamento das convulsões quer no tratamento do delirium, a utilização de benzodiazepinas como fármacos de primeira linha, administradas preferencialmente por via parentérica. Nos casos em que o quadro clínico de delirium inclua a presença de alucinações e os doentes não se encontrem adequadamente estabilizados apenas com benzodiazepinas, está recomendada a prescrição de antipsicóticos. $^{20}$

Segundo as guidelines da WFSBP, os fármacos de primeira linha para tratamento da síndrome de abstinência alcoólicasão as benzodiazepinas. As benzodiazepinas mais frequentemente utilizadas são diazepam, clordiazepóxido, oxazepam, lorazepam e alprazolam. A dose indicada de benzodiazepina a prescrever depende da gravidade da PUA, da intensidade da síndrome de abstinência alcoólica e também das características individuais do doente. ${ }^{9}$

As guidelines da WFSBP referem a existência de evidência preliminar sobre a utilidade de uso de lamotrigina, memantina e topiramato no tratamento da síndrome de abstinência alcoólica (recomendação de nível C para o topiramato e recomendação de nível $D$ para a lamotrigina e memantina). É também referido que a carbamazepina e a oxcarbamazepina revelaram eficácia no tratamento da síndrome de abstinência alcoólica de intensidade moderada a grave, e que o ácido valpróico revelou eficácia apenas no tratamento da síndrome de abstinência alcoólica de intensidade moderada. Tanto a carbamazepina como o ácido valpróico estão contraindicados em doentes com patologia hepática e hematológica. ${ }^{9}$

Em relação ao uso de agonistas dos receptores alfa-2 adrenérgicos, como a clonidina, as guidelines da WFSBP recomendam a sua prescrição apenas em situações em que os sinais de hiperatividade adrenérgica sejam de intensidade grave. ${ }^{9}$

As guidelines da WFSBP recomendam a administração profilática de tiamina por via parentérica a doentes com história de dependência de álcool ou com sinais ou sintomas sugestivos de síndrome de Wernicke-Korsakoff. ${ }^{9}$ No delirium por privação de álcool, recomendam a prescrição de benzodiazepinas. ${ }^{9}$

As mais recentes guidelines da American Psychiatric
Association (APA) relativas ao tratamento das PUA (2018) não abordam a componente farmacológica da fase de desintoxicação de álcool, apenas fazem uma análise dos psicofármacos recomendados para o tratamento da fase de manutenção de abstinência. ${ }^{24}$ Há, porém, uma referência ao assunto nessas guidelines, na seção das benzodiazepinas, onde vem referido que estas são os fármacos de primeira linha no tratamento da síndrome de abstinência alcoólica. No entanto, as guidelines da APA de 2006, que abordam o tratamento farmacológico da síndrome de abstinência alcoólica, recomendam para tratamento desta síndrome o uso de benzodiazepinas, sendo as mais frequentemente prescritas o diazepam, oxazepam e lorazepam. Os beta-bloqueantes, a clonidina, anticonvulsivantes e antipsicóticos são recomendados para utilizar associados às benzodiazepinas, em caso de necessidade, mas não estão recomendados para uso em monoterapia, dado não terem apresentado eficácia na prevenção nem de convulsões nem de delirium por privação de álcool. ${ }^{25}$

Relativamente à utilização de anticonvulsivantes, as guidelines da APA referem que se tem demonstrado eficácia semelhante entre a carbamazepina e as benzodiazepinas para prevenção das convulsões por privação de álcool, contudo, não recomendam a utilização de fenitoína com esse propósito, excepto se já for a medicação de ambulatório habitualmente realizada num doente com diagnóstico de epilepsia que tenha suspendido a toma de terapêutica. A utilização de carbamazepina está recomendada sobretudo para síndromes de abstinência alcoólica de intensidade ligeira a moderada. ${ }^{25}$

Nos doentes com delirium, delírios ou alucinações, as guidelines da APA de 2006 recomendam a utilização de fármacos antipsicóticos, em particular, o haloperidol. No entanto, recomendam que se use em associação à prescrição de benzodiazepinas, já que os fármacos antipsicóticos não são os responsáveis pelo tratamento e remissão da sintomatologia referida. ${ }^{25}$

As principais recomendações das guidelines da NICE, ASAM, WFSBP e APA encontram-se resumidas na Tabela 1.

\section{DISCUSSÃO}

Assim, do conjunto das quatro guidelines principais relativas ao tratamento farmacológico recomendado para prevenção e tratamento da síndrome de abstinência alcoólica, podemos concluir que:

- Os fármacos de primeira linha recomendados em todas as guidelines para a prevenção e tratamento da síndrome de abstinência alcoólica são as as benzodiazepinas.

- As principais benzodiazepinas descritas na literatura como sendo utilizadas para estas situações são o diazepam, oxazepam, lorazepam, alprazolam e clorodiazepóxido, apesar de não estar demonstrada uma eficácia superior de nenhuma benzodiazepina sobre as restantes na prevenção e tratamento da síndrome de abstinência alcoólica. 
Tabela 1 - Principais recomendações das guidelines da NICE, ASAM, WFSBP e APA

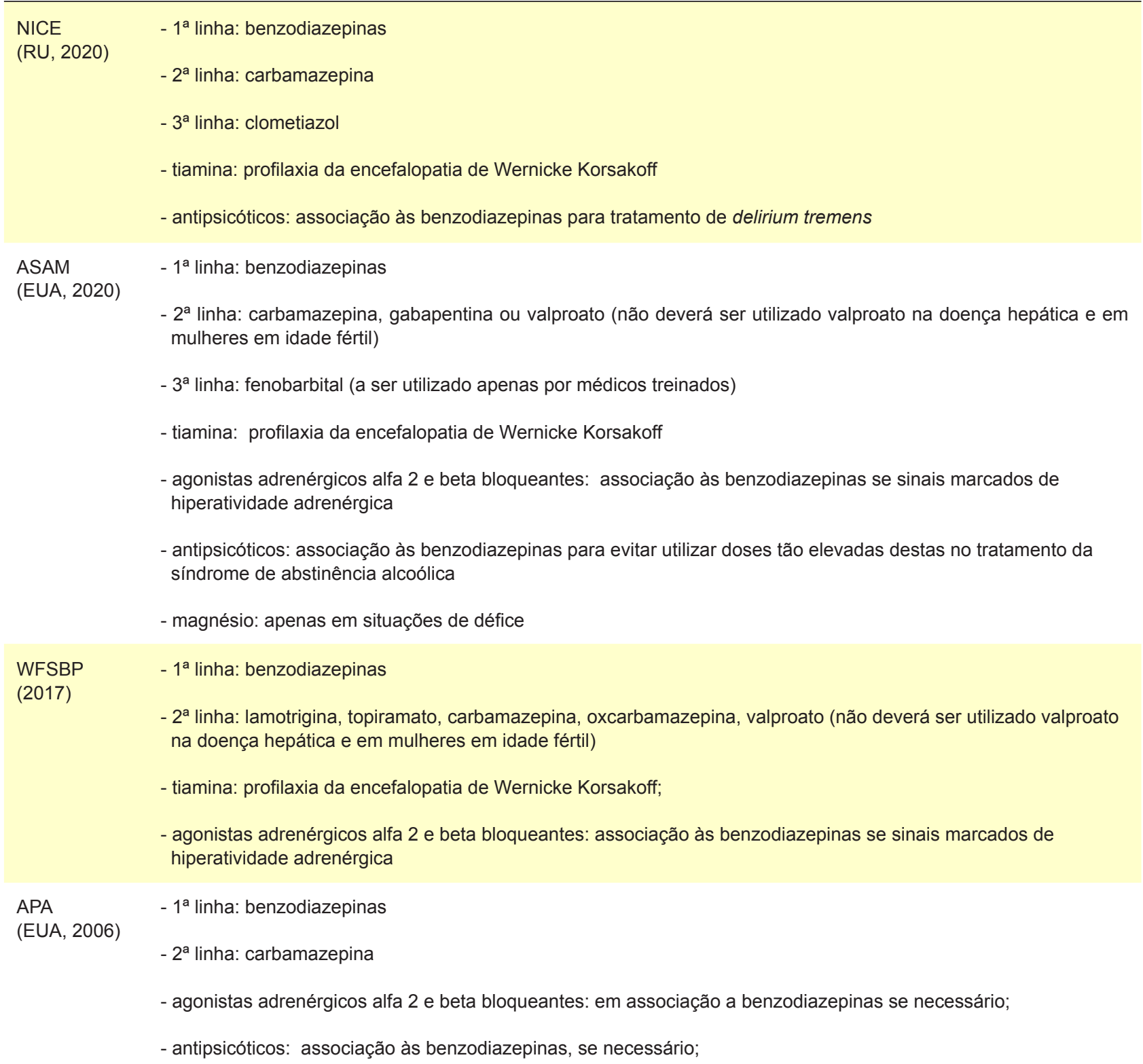

NICE: National Institute for Health and Care Excellence; ASAM: American Society of Addiction Medicine; WFSBP: World Federation of Societies of Biological Psychiatry; APA: American Psychiatric Association; RU: Reino Unido; EUA: Estados Unidos da América

- A dosagem de benzodiazepinas a prescrever deve ser calculada de acordo com a gravidade da PUA, a intensidade da síndrome de abstinência alcoólica e as características individuais do doente, devendo ser ajustada em doentes idosos ou com doença hepática.

- A prescrição de carbamazepina está recomendada nas quatro guidelines como alternativa ao uso de benzodiazepinas quando estas estão contraindicadas; no entanto, a maioria destas guidelines refere carbamazepina como uma alternativa possível às benzodiazepinas, mas apenas para síndromes de abstinência alcoólica de intensidade ligeira a moderada.

- A recomendação do uso de anticonvulsivantes é consensual em todas as guidelines, nomeadamente a carbamazepina e o valproato, como fármacos a prescrever em associação às benzodiazepinas com o propósito de reduzir a dose de utilização das mesmas. Porém, está contraindicada a utilização de fenitoína, e a prescrição de carbamazepina e valproato deve ser feita com precaução em doentes com patologia hepática, hematológica, ou em mulheres em idade fértil.

- A maioria das guidelines recomenda a prescrição de tiamina de forma profilática a todos os doentes, para prevenir o desenvolvimento da encefalopatia de Wernicke-Korsakoff.

- A via de administração recomendada de tiamina é, numa fase inicial, a via parentérica, podendo ser utilizada posteriormente a via oral.

- A prescrição de magnésio está recomendada 
apenas em doentes com hipomagnesiémia, arritmias cardíacas, desequilíbrios hidro-electrolíticos ou em doentes com antecedentes de convulsões em contexto de privação de álcool.

- Em situações de desequilíbrio hidro-eletrolítico, nomeadamente défices em ácido fólico, magnésio, fósforo e potássio, deverá proceder-se à correção desses défices.

- A prescrição de agonistas adrenérgicos alfa-2 e de betabloqueantes deve ser realizada apenas em combinação com a prescrição de benzodiazepinas e em doentes com sinais de hiperatividade autonómica que sejam de intensidade marcada.

- Em caso de ocorrência de convulsões por privação de álcool, o tratamento recomendado de primeira linha são as benzodiazepinas.

- Em caso de ocorrência de delirium tremens, o tratamento recomendado de primeira linha são as

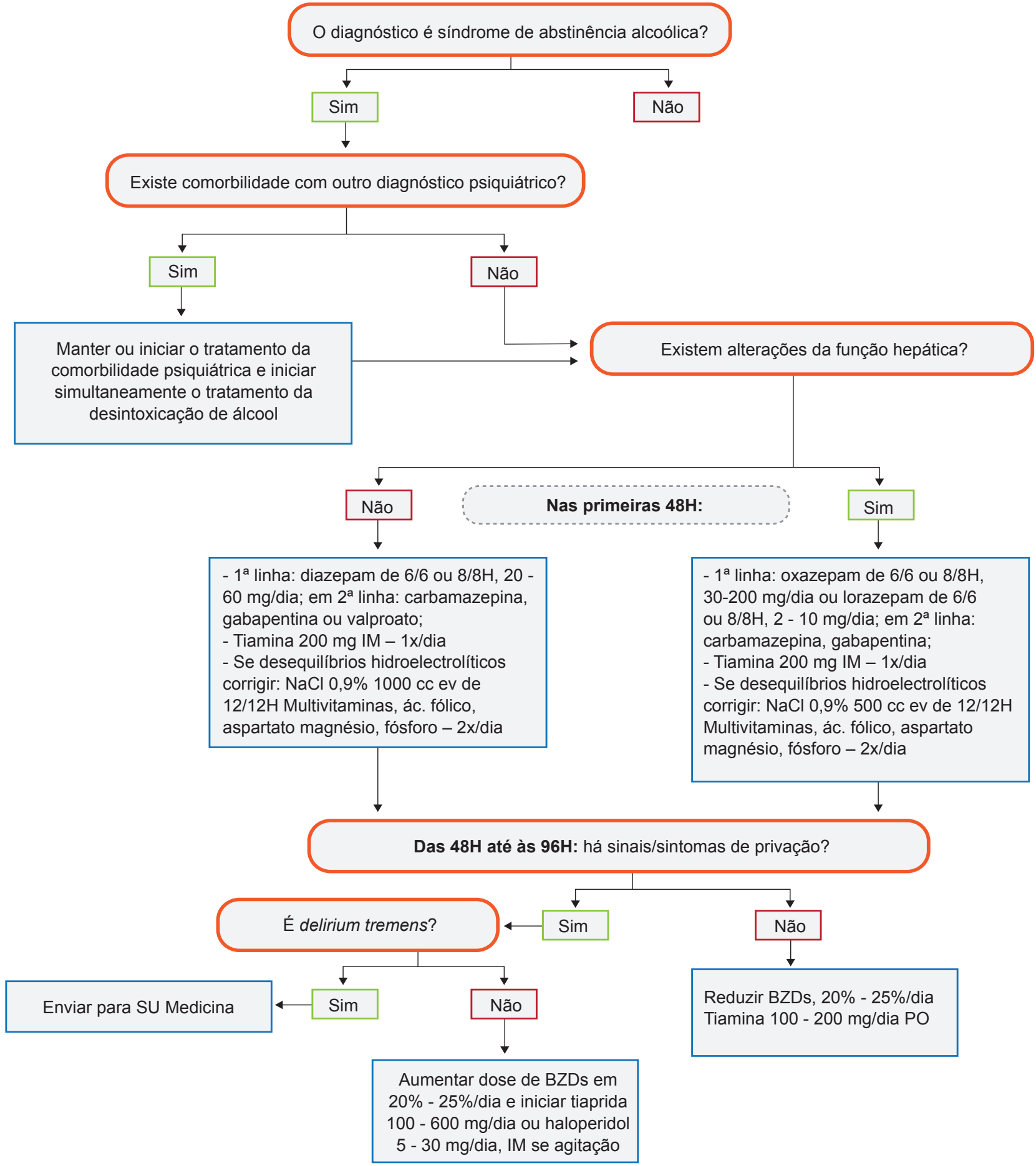

Figura 1 - Tratamento farmacológico para síndrome de privação de álcool

SU: serviço de urgência; BZDs: benzodiazepinas; DHC: doença hepática crónica; IH: insuficiência hepática; PO: per os; IM: intramuscular 
benzodiazepinas, podendo ser associadas ou não à prescrição de antipsicóticos.

De acordo com as guidelines de Maudsley, ${ }^{5}$ a duração recomendada para realização do tratamento farmacológico para a síndrome de abstinência de álcool é de sete a 10 dias. Nesse período, a dose de benzodiazepinas deverá ser progressivamente reduzida, em cerca de $20 \%$ a $25 \%$ por dia, de modo a, idealmente, terminar o tratamento de desabituação física já sem prescrição de benzodiazepinas.

Na presença de agitação psicomotora, deverá ser considerado o diagnóstico de delirium tremens. Na sua exclusão deve proceder-se à revisão da dose de benzodiazepinas prescritas e, caso esta medida não seja suficiente, deverá ser considerada a prescrição de terapêutica intramuscular para controlo da agitação psicomotora (haloperidol ou tiaprida) conforme recomendado nas guidelines., 56

Em doentes que necessitam de tratamento para a síndrome de abstinência alcoólica é importante avaliar a presença de comorbilidade psiquiátrica. Está comprovada uma elevada taxa de comorbilidade entre PUA e patologia psiquiátrica pelo que, na presença de patologia psiquiátrica em fase de descompensação aguda, esta deve ser tratada simultaneamente com o tratamento a realizar para a síndrome de abstinência alcoólica. ${ }^{27,28}$

Ao analisar o conjunto destas recomendações, e considerando a sua aplicabilidade à realidade nacional, apresenta-se de seguida um fluxograma que resume o tratamento farmacológico recomendado da síndrome de abstinência alcoólica (Fig. 1).

\section{REFERÊNCIAS}

1. Serviço de Intervenção nos Comportamentos Aditivos e nas Dependências. Relatório Anual 2018: a situação do país em matéria de álcool. 2018. [consultado 2020 nov 25]. Disponível em: http://www.sicad. pt/PT/Documents/2019/relatorio_2018/RelatorioAnual_2018_\%20 ASituacaoDoPaisEmMateriaDeAlcool.pdf.

2. Ministério da Saúde. Retrato da Saúde, Portugal; 2018. [consultado 2020 nov 20]. Disponível em: https://www.sns.gov.pt/wp-content/ uploads/2018/04/retrato-da-saude_2018_compressed.pdf.

3. World Health Organization. Global status report on alcohol and health 2018. 2018. [consultado 2020 nov 25]. Disponível em: https://www.who. int/substance_abuse/publications/global_alcohol_report/gsr_2018/en/.

4. Costa L, Teixeira J. Critérios de referenciação para consulta de alcoologia pelos Cuidados de Saúde Primários. Acta Med Port. 2021;34:160.

5. Taylor D, Barnes T, Young A. The Maudsley prescribing guidelines in psychiatry. $13^{\text {th }}$ ed. Hoboken: John Wiley \& Sons; 2018.

6. Latifi S, MesserT. The efficacy of tiapride and carbamazepine combination therapy in reducing alcohol withdrawal symptoms: a systematic review and meta-analysis. Pharmacopsychiatry. 2019;52:209-16.

7. American Psychiatric Association. DSM-5 - Manual de Diagnóstico e Estatística das Perturbações Mentais. $5^{\text {th }}$ ed. Forte da Casa: Climepsi; 2014.

8. World Health Organization. CID-10 - Classificação de Transtornos Mentais e de Comportamento da CID- 10. Porto Alegre: Artmed; 2000.

9. Soka M, Kranzler H, Hesselbrock V, Kasper S, Mutschler J, Möller HJ. WFSBP Task Force on Treatment Guidelines for Substance Use Disorders. Guidelines for biological treatment of substance use and related disorders, part 1: alcoholism, first revision. World $\mathrm{J}$ Biol Psychiatry. 2017;18:86-119.

10. Hansen HC, Maschke M, Schuchardt V, Tiecks F. Alcohol delirium. In: Diener HC, Putzki N, Berlit P, editors. Guidelines for Diagnosis and Therapy in Neurology. New York: Stuttgart Thieme; 2005. p. 448-54.

11. Brust J. Acute withdrawal: diagnosis and treatment. Handb Clin Neurol. 2014;125:123-31.

12. Curran G, Sullivan G, Williams K, Han X, Allee E, Kotrla K. The association

\section{CONCLUSÃO}

A elevada prevalência de PUA em Portugal, associada ao recurso muito frequente de doentes com PUA aos serviços de Urgência por causas com ela direta ou indiretamente relacionadas, torna muito provável o contacto de médicos das várias especialidades médico-cirúrgicas com a síndrome de abstinência alcoólica.

Podemos assim concluir que é fundamental manter um elevado nível de suspeita para o diagnóstico da síndrome de abstinência alcoólical, de modo a detetar precocemente esses casos e iniciar de imediato o tratamento farmacológico mais adequado.

Esta revisão contribui para colmatar a escassez de normas de orientação clínica em Portugal relativas ao tratamento farmacológico da síndrome de abstinência alcoólica. As guidelines internacionais disponíveis sobre este reúnem consenso nos princípios básicos gerais do tratamento, e todas apresentam informação que é relevante em determinados aspetos, sendo, contudo, omissas noutros.

\section{CONFLITOS DE INTERESSE}

A autora declara não ter qualquer conflito de interesse relativamente ao presente artigo.

\section{FONTES DE FINANCIAMENTO}

Não foi utilizada nenhuma bolsa ou subsídio para a realização do trabalho.

of psychiatric comorbidity and use of the emergency department among persons with substance use disorders: an observational cohort study. BMC Emerg Med. 2008;8:17.

13. Sanjuan P, Rice S, Witkiewitz K, Mandler R, Crandall C, Bogenschutz MP. Alcohol, tobacco, and drug use among emergency department patients. Drug Alcohol Depend. 2014;138:32-8.

14. Sarfraz M, Landron S, Klugman A. Alcohol abuse among English and French psychiatric referrals from accident and emergency departments. Alcohol Alcohol. 2008;43:589.

15. te Wildt B, Andreis C, Auffahrt I, Tettenborn C, Kropp S, Ohlmeier M. Alcohol related conditions represent a major psychiatric problem in emergency departments. Emerg Med J. 2006;23:428-30.

16. Wu L, Swartz M, Wu Z, Mannelli P, Yang C, Blazer D. Alcohol and drug use disorders among adults in emergency department settings in the United States. Ann Emerg Med. 2012;60:172-80.e5.

17. National Institute for Health and Care Excellence. Alcohol-use disorders: diagnosis and management of physical complications; 2010. [consultado 2020 nov 25]. Disponível em: http://www.nice.org.uk/guidance/cg100.

18. National Institute for Health and Care Excellence. Alcohol-use disorders: diagnosis and management of physical complications; 2017. [consultado 2020 nov 25]. Disponível em: www.nice.org.uk/guidance/ cg100/resources/alcoholuse-disorders-diagnosis-and-management-ofphysical-complications-pdf-35109322251973.

19. National Institute for Health and Care Excellence. Assisted alcohol withdrawal; 2020. [consultado 2020 nov 25]. Disponível em: https:// pathways.nice.org.uk/pathways/alcohol-use-disorders/assisted-alcoholwithdrawal\#content=view-pathways-A-to-Z.

20. American Society of Addiction Medicine. The ASAM Clinical Practice Guideline on Alcohol Withdrawal Management; 2020. [consultado 2020 nov 25]. Disponível em: http://www.asam.org/docs/default-source/ quality-science/the_asam_clinical_practice_guideline_on_alcohol-1. pdf?sfvrsn=ba255c2_2.

21. Sullivan, JT, Sykora K, Schneiderman J, Naranjo CA, Sellers EM. Assessment of alcohol withdrawal: The revised Clinical Institute 
Withdrawal Assessment for Alcohol scale (CIWA-Ar). $\mathrm{Br} \mathrm{J}$ Addict. 1989;84:1353-17.

22. Sarai M, Tejani A, Chan A, Kuo I, Li J. Magnesium for alcohol withdrawal. Cochrane Database Syst Rev. 2013;6:CD008358.

23. Teixeira J, Mota T, Fernandes J. Nutritional evaluation of alcoholic inpatients admitted for alcohol detoxification. Alcohol Alcohol. 2011;46:558-60.

24. Reus V, Fochtmann L, Bukstein O, Eyler A, Hilty D, Horvitz-Lennon M, et al. The American Psychiatric Association Practice guideline for the pharmacological treatment of patients with alcohol use disorder. Am J Psychiatry. 2018;175:86-90.

25. American Psychiatric Association. Practice guideline for the treatment of patients with substance use disorders. 2006. [consultado 2020 nov 20]. Disponivel em: https://www.lyouthcare.com/content/dam/centene/ illinois/pdfs/substanceuse_practice\%20guideline.pdf.

26. Allain H, Dautzenberg P, Maurer K, Schuck S, Bonhomme D, Gerard D. Double blind study of tiapride versus haloperidol and placebo in agitation and aggressiveness in elderly patients with cognitive impairment. Psychopharmacology. 2000;148:361-6.

27. DervauxA, LaqueilleX. Psychiatric comorbidities in alcohol dependence. Press Med. 2018;47:575-85.

28. Pettinati $\mathrm{H}$, O'Brien $\mathrm{C}$, Dundon W. Current status of co-occurring mood and substance use disorders: a new therapeutic target. Am J Psychiatry. 2013;170:23-30. 